\title{
Labyrinthe
}

25 | 2006 (3)

La Bande dessinée : ce qu'elle dit, ce qu'elle montre

\section{Le voyage ménippéen et les limites du savoir humain}

La modeste philosophie d'un genre antiphilosophique, de la Renaissance aux Lumières

\section{Nicolas Corréard}

\section{(2) OpenEdition}

Journals

Édition électronique

URL : http://journals.openedition.org/labyrinthe/1420

DOI : $10.4000 /$ labyrinthe. 1420

ISSN : 1950-6031

Éditeur

Hermann

Édition imprimée

Date de publication : 1 décembre 2006

Pagination : 121-125

ISBN : 2-9526131-2-5

Référence électronique

Nicolas Corréard, «Le voyage ménippéen et les limites du savoir humain », Labyrinthe [En ligne],

25 | 2006 (3), mis en ligne le 28 mars 2010, consulté le 20 avril 2019. URL : http://

journals.openedition.org/labyrinthe/1420; DOI : 10.4000/labyrinthe.1420

Ce document a été généré automatiquement le 20 avril 2019.

Propriété intellectuelle 


\section{Le voyage ménippéen et les limites du savoir humain}

La modeste philosophie d'un genre antiphilosophique, de la Renaissance aux Lumières

Nicolas Corréard

1 D'Alberti à Voltaire, l'Europe littéraire s'est passionnée pour les œuvres de l'écrivain grec Lucien de Samosate (IIe siècle après J.-C.), notamment pour ses récits des aventures du personnage de Menippus: parti en quête de la sagesse, Menippus consulte les plus illustres représentants des écoles philosophiques de l'Antiquité ${ }^{\text {. Seulement, leurs }}$ discours se contredisent. L'apprenti philosophe décide alors d'aller voir par lui-même les arcanes des enfers (dans la Necyomantia) ou du ciel (dans l'Icaromenippus), mais le voyage ne lui apprend rien d'autre que ce qu'il savait déjà : que la spéculation théorétique est vaine, et qu'il faut se contenter d'une vie ordinaire. Au nom du sens commun, Lucien se moquait du désir, consubstantiel à la philosophie antique, d'acquérir un savoir au-delà de l'opinion. De réécritures en re-créations, le récit ménippéen, que nous définirons comme le récit satirique d'une quête intellectuelle infructueuse, donne lieu à des avatars dans toutes les langues européennes à la Renaissance ${ }^{2}$. Une approche comparatiste permet de prendre la mesure de ce vaste corpus caractérisé à la fois par la reprise de «lieux » identiques, et par la variété de leurs agencements possibles: le désir de connaissance initial, motivé par la «curiosité» ou la "perplexité»; la consultation des autorités savantes; l'expérience de la contradiction des théories entre elles, qui traduit leur absence de fondement; la mise en scène fictionnalisante de l'enquête, dont les moyens sont tout aussi improbables que les buts; la déception finale devant son caractère non conclusif. Souvent, les disciplines des savoirs modernes remplacent celles de l'Antiquité, et les récits ménippéens des XVIe et XVIIe siècles deviennent le lieu d'une débauche d'érudition, sous la forme d'une revue critique.

Dès lors, une perspective strictement littéraire, à l'instar des études existantes sur le lucianisme, ne suffit pas à rendre compte de l'histoire des fictions ménippéennes, ni à expliquer le penchant pour la satire antiphilosophique et anti-intellectuelle à laquelle elles donnent lieu. Celle-ci ne prend sens qu'en étroite corrélation avec l'histoire des 
idées : la quête fictionnelle d'une vérité inexistante a servi de mode d'expression d'une incertitude vécue comme indépassable par les auteurs, dans le contexte d'une crise épistémologique durable et de grande ampleur, affectant tous les champs du savoir ${ }^{3}$. La vogue du récit ménippéen a ainsi doublé la redécouverte et la diffusion des idées sceptiques de diverses obédiences, au sens où elle n'a cessé d'accompagner leur rejet des autorités et des paradigmes établis, lui donnant voix dans une forme imaginative, jouant de la critique de l'incertitude des sciences pour valoriser a contrario l'écriture non savante de la fiction. Doublé, aussi, au sens où elle dit autre chose : il s'agit moins de discuter l'existence ou l'absence d'un critère de vérité que de constater la fondamentale irrationalité de la condition humaine, l'impensé de tout savoir. Doublé, enfin, au sens où cette vogue du lucianisme a précédé historiquement l'essor d'argumentations proprement philosophiques du scepticisme moderne, tout comme elle a survécu aux diverses réfutations du doute radical dont elle se nourrit, élaborées par les grands penseurs rationalistes à partir du milieu du XVIIe siècle.

Quand Leon Battista Alberti ressuscite la fiction lucianesque avec son Momus (vers 1450), il exprime déjà un désarroi profond, aux antipodes de l'image d'Épinal d'un humanisme optimiste entonnant sans bémol l'éloge de la connaissance : les philosophes dérisoires qui s'agitent à la surface de la terre, décrits selon les stéréotypes véhiculés par les écrits doxographiques et par Lucien lui-même (Platon dans ses rêves, Diogène dans son tonneau, Socrate ergoteur, Démocrite rieur, etc.), tombent sous le coup de la condamnation chrétienne de la curiosité. Ces géants qui voulaient voler le feu au ciel ont présumé des forces de la raison humaine, à laquelle les secrets de la nature resteront toujours inaccessibles. Mais dans l'esprit d'Alberti, les figures des Anciens représentent aussi les modernes, les humanistes eux-mêmes, engagés dans un projet de connaissance universelle qui échoue, dans les faits, à s'affranchir de la relativité des choses de ce monde. Les multiples traductions des œuvres complètes de Lucien au XVIe siècle (du grec au latin, puis en vulgaire), éditées en Europe du Sud comme en Europe du Nord, nourrissent parallèlement l'intérêt pour des formes lucianesques telles que l'éloge paradoxal, qui connaît son apogée avec l'Éloge de la folie d'Érasme (1511) et le traité De l'incertitude et de la vanité des sciences de Corneille Agrippa (1527).

Le récit ménippéen devient alors le moyen de moquer une libido sciendi désireuse de transgresser les limites métaphysiques de la connaissance, voire le moyen de prôner l'ignorance heureuse contre l'étude, coûteuse et inutile. Ainsi des récits facétieux d'Anton Francesco Doni, notamment I mondi e gli inferni (1552): les voyages fantastiques des Académiciens dans les cieux sont l'occasion de vérifier la nullité de leur science, tandis qu'ils traversent des enfers peuplés de pédants qui « s'alambiquent la cervelle » pour rien. Cette œuvre soutient la comparaison avec Rabelais, dont le Tiers Livre (1545), sans doute le rejeton le plus inspiré et le plus original du genre, explore autrement ce que Doni appelle les «fictions issues du savoir humain». Plus truculent, étant donné les motifs de l'enquête de Panurge (se mariera-t-il ? sera-t-il cocu ?), le récit de Rabelais est aussi plus subtil : les paroles des savants sont incertaines car ambiguës, elles en disent à la fois trop (elles prennent la forme de logorrhées sans portée pratique) et trop peu (elles ressassent des évidences). L'expérience de Panurge traduit un motif récurrent des œuvres lucianesques: « Plus on a de savoir, et plus on a de peine. "

5 À l'origine affilié au cynisme par la figure de Menippus, le récit lucianesque devient donc le lieu d'une critique radicale de la possibilité et/ou de la valeur de la connaissance, qui à l'époque moderne emprunte plus encore aux idées sceptiques, certains auteurs allant 
jusqu'à mettre en scène leur inclination pour des positions suspensives. Le plus emblématique est sans doute l'Espagnol Saavedra Fajardo, proche de Quevedo et de Gracián, mais aux sympathies pyrrhoniennes affichées. Sa República literaria (v. 1630) est une critique extensive des connaissances de l'époque, sous la forme du voyage satirique dans la République des lettres. Aucune discipline n'échappe à l'incertitude, pas plus les sciences de la nature (astronomie, cosmologie, physique, etc.) que celles qui prennent l'homme pour objet (droit, médecine, etc.). L'histoire fait elle aussi figure de cible privilégiée : concurrents discrédités des récits de fictions, les récits des historiens sont victimes de la fausseté de leurs informations et de la partialité de leurs auteurs. L'encyclopédisme inversé de ces textes n'exclut pas toujours, pour finir, la théologie. Un auteur érasmisant comme Villalón (Crotalón, 1555) amplifiait déjà les récits lucianesques en pointant, sous les contradictions des " philosophes ", celles des scolastiques. Au temps des guerres de religion, les débats théologiques offrent le spectacle d'une dissension permanente facile à mettre en scène par le biais du topos lucianesque de la dispute confuse et ridicule des théoriciens. Les injonctions bibliques de l'Ecclésiaste et de saint Paul, qui pourfendent la prétention à une sagesse humaine et qui recommandent de borner la curiosité, se voient paradoxalement convoquées dans ces textes par des voix incrédules, parfois même agnostiques, qui s'en prennent à ceux qu'un lucianiste français, Tahureau, nomme les « importuns scrutateurs des choses douteuses ». Au plus fort d'une controverse qui déchire son pays, un humaniste hollandais, Petrus Cunaeus, raconte ainsi un voyage dans une île de lettrés devenus fous, où la Sagesse intervient en personne pour faire taire les disputes : elle annonce qu'elle ne révélera jamais ses secrets, et vante en conséquence l'attitude tolérante des sceptiques, qui suspendent leur jugement sur les questions métaphysiques (Sardi Venales, 1612).

6 Parent du récit picaresque à l'espagnole et de l'histoire comique à la française, qui relèguent l'érudition au second plan, le récit ménippéen ne s'efface qu'en apparence au temps des Lumières. Quand le Gulliver de Swift découvre les aberrations des savants de Laputa au livre III de ses Voyages (1726), et quand Sterne place au cœur de Tristram Shandy (1760) l'extraordinaire "récit de Slawkenbergius", où les raisonneurs de tout poil s'acharnent à saisir une vérité plus que jamais cachée « au fond du puits ", ils appliquent les codes du genre à des cibles d'actualité : ce sont maintenant les partisans de la science nouvelle, les faiseurs de systèmes philosophiques (de Descartes à Leibniz), ou les enthousiastes modernes certains de connaître la divinité. On pourra reconsidérer à cette aune la production voltairienne des « contes». Leur auteur a pu faire l'éloge du progrès historique de la raison, mais dans un sens bien plus restreint qu'on ne le croit ; il s'est aussi avoué "philosophe ignorant", qui ressasse la faiblesse et l'incapacité de l'esprit humain, et recourt en conséquence au récit ménippéen. C'est dans les moins connus de ses contes que la dette est la plus flagrante, par exemple L'Homme aux quarante écus (1768), où la traversée des grandes questions intellectuelles du jour aboutit à la seule conclusion possible, «douter de tout». Micromégas et Candide seront les derniers enfants de Ménippe, avant que le genre ne se dissolve dans le roman de formation moderne, se manifestant par intermittences (qu'on pense à l'antipositivisme de Bouvard et Pécuchet, que Flaubert écrit avec Lucien et Rabelais sous la main).

Rétrospectivement, cet effacement montre à quel point son destin était lié à un contexte intellectuel de prévalence de l'incertitude : au seuil de la modernité, le récit ménippéen a pu constituer un moyen alternatif, plus divertissant peut-être, mais tout aussi efficace, sinon plus, que des genres sérieux comme l'essai ou le traité, pour formaliser l'expérience 
d'une crise épistémologique. Un moyen logique en tout cas, car le passage à la fiction permet de rendre compte des impasses du discours savant en se plaçant en dehors, ou audelà : l'auteur du récit se trouve exempté du scepticisme qu'il exprime, car il ne tient pas, pour sa part, de discours assertif ou thétique. C'est bien la question du statut de la fiction dans l'économie des discours qui est posée, sur le plan théorique comme sur le plan historique, par le genre du récit ménippéen.

\section{NOTES}

1. Cette recherche constitue le premier volet d'une thèse de doctorat de littérature comparée portant sur « Les fictions du scepticisme en Europe : 1540-1660», sous la direction de F. Lavocat (Paris VII).

2. Pour obtenir une cartographie complète de l'influence considérable de Lucien, il faut mettre bout à bout les travaux de Ch. Robinson, Lucian and his Influence in Europe, 1979; E. Mattioli, Luciano e l'Umanesimo, 1980 (sur l'Italie); Ch. Lauvergnat-Gagnière, Lucien de Samosate et le lucianisme en France au XVIe siècle, 1988 (sur la France) ; M. Zappala, Lucian of Samosata in the Two Hesperias, 1990 (sur l'Espagne).

3. L'Histoire du scepticisme d'Érasme à Spinoza de R. Popkin (Puf, «Léviathan », 1995) reste sans doute la meilleure introduction à ce sujet. Ce vaste panorama appelle cependant la discussion, ne serait-ce que parce qu'il réduit la question du scepticisme moderne à celle de la transmission et de la discussion des arguments hérités du pyrrhonisme antique. Ce faisant, il laisse échapper l'essentielle polymorphie du phénomène sceptique aux XVIe et XVIIe siècles.

\section{AUTEUR}

\section{NICOLAS CORRÉARD}

Correardnicolas[at]hotmail.com 